\title{
Intrauterine device and cervical cancer: we need more evidence
}

\author{
Cristina López del Burgo ${ }^{\mathrm{a}, \mathrm{c}^{*}}$ \\ Alfonso Osorio ${ }^{\text {b,c }}$ \\ Jokin de Irala ${ }^{\mathrm{a}, \mathrm{c}}$
}

${ }^{a}$ Department of Preventive Medicine and Public Health,

Faculty of Medicine, University of Navarra, 31080 Pamplona, Spain

${ }^{\mathrm{b}}$ Department of Education, University of Navarra, 31080 Pamplona, Spain

${ }^{\mathrm{c}}$ Institute for Culture and Society, University of Navarra, 31080 Pamplona, Spain

*Corresponding author:

Tel: +34948425600 (ext. 6360) Fax: +34 948425649

E-mail address: cldelburgo@unav.es

Published in:
The Lancet Oncology
(www.thelancet.com)

See original article in Journal's web page:

http://www.thelancet.com/journals/lanonc/article/PIIS1470-2045\%2811\%2970354-

$\underline{0 / \text { fulltext }}$

\section{Cite as:}

López-del Burgo C, Osorio A \& De Irala J. Intrauterine device and cervical cancer: we need more evidence. The Lancet Oncology 2011;12:1185-1186. 
In their study on Intrauterine device use (IUD) and risk of cervical cancer published in The Lancet Oncology, Xavier Castellsagué and colleagues ${ }^{1}$ claimed that their work provides evidence that IUD use might reduce the risk of developing cervical to solid evidence that IUD use might reduce the risk of developing cervical cancer.

The authors pursued alternative explanations using stratified analyses with possible confounders and investigated plausible mechanisms for the reported effect. They were right to have done so, not only for scientific reasons but also because studies such as these are rapidly picked up by the media, who sometimes draw premature or unrealistic conclusions from such data. Additionally, this study has important commercial implications; therefore, caution in the interpretation of these results is necessary. We would like to put forward some ideas in this letter that might help to understand the results better.

Our main concern is related to the ever/never classification of exposure variables. Although some of the crude analyses are presented with IUD use as a categorical variable, the main results are estimated and presented with ever/never use.

Oral Contraceptive use is clearly related to cervical cancer ${ }^{2}$ and therefore its inclusion in the main analysis would have yielded better adjusted results. However, oral contraceptive use cannot be evaluated precisely without simultaneously taking duration of exposure and timing of exposure into account, because induction and latency periods are key issues in cancer epidemiology. ${ }^{3}$ Therefore, the ever/never stratification of oral contraceptive use is probably not precise enough to adjust for confounding. A lower oral contraceptive use in long term IUD users than in short-term IUD users or those who have never used an IUD cannot be completely ruled out as an alternative explanation for the lower cervical cancer risk seen in long-term IUD users.

When data were stratified by duration of oral contraceptive exposure $(0,1,2-4,5-9$, and $\geq 10$ years; presented in the web appendix $\left.{ }^{1}\right)$, most of the associations were not significant, and there was even one positive association for IUD users that used oral contraceptives for 2-4 years, which was not discussed in the main Article.

Furthermore, the associations between IUD use and cervical cancer in those who have never used oral contraceptives are not adjusted for other important confounders, such as alternative family planning methods used by those not using IUDs (eg, other hormonal methods that are more prevalent in developing countries), number of sexual partners, and smoking.

The imprecise measurement of a crucial confounder such as oral contraceptive use is the main weakness of this study. A better defined reference category of exposure would have been much more informative. 


\section{References}

1 Castellsague X, Diaz M, Vaccarella S, et al. Intrauterine device use, cervical infection with human papillomavirus, and risk of cervical cancer: a pooled analysis of 26 epidemiological studies. Lacet Oncol. 2011; (published online Sept 13. DOI:10.1016/S1470-2045(11)70223-6.).

2 Appleby P, Beral V, Berrington de Gonzalez A, et al. Cervical cancer and hormonal contraceptives: collaborative reanalysis of individual data for 16,573 women with cervical cancer and 35,509 women without cervical cancer from 24 epidemiological studies. Lancet. 2007; 370 (9599): 1609-21.

3 Bosch FX, Lorincz A, Munoz N, Meijer CJ, Shah KV. The causal relation between human papillomavirus and cervical cancer. J Clin Pathol. 2002; 55(4): 244-65. 